\title{
Metastable legged locomotion: methods to quantify and optimize reliability
}

\author{
Cenk Oguz Saglam and Katie Byl \\ Electrical and Computer Engineering Department, UC Santa Barbara, CA 93106 USA
}

\begin{abstract}
Measuring the stability of highly-dynamic bipedal locomotion is a challenging but essential task for more capable human-like walking. By discretizing the walking dynamics, we treat the system as a Markov chain, which lends itself to an easy quantification of failure rates by the expected number of steps before falling. This meaningful and intuitive metric is then used for optimizing and benchmarking given controllers. While this method is applicable to any controller scheme, we illustrate the results with two case demonstrations. One scheme is the now-familiar hybrid zero dynamics approach and the other is a method using piece-wise reference trajectories with a sliding mode control. We optimize low-level controllers, to minimize failure rates for any one gait, and we adopt a hierarchical control structure to switch among low-level gaits, providing even more dramatic improvements on the system performance.
\end{abstract}

\section{INTRODUCTION}

Locomotion is an undeniable necessity for many robot applications. Bipedal robot walking is an anthropomorphic approach, providing intuitive means for negotiating intermittent or otherwise rough terrain, where wheels would be ineffective. Toward developing more capable two-legged robots, measuring performance of legged locomotion is essential. As stated in Hobbelen, ${ }^{1}$ performance of highly-dynamic walkers is often measured by speed or energy consumption which are secondary metrics to the reliability of walking operation. However, robustness needs to be quantified to achieve "good" disturbance rejection and operate reliably in real-world environments. In this work we quantify and optimize control for robustness to terrain disturbance, or equivalently, for stability on rough terrain.

As in many zero-moment point approaches, stability is often conservatively defined as a binary metric to avoid the rotation of the stance foot, to ensure not-falling. ${ }^{2}$ However, robust, dynamic, fast, agile, and energy efficient human walking exploits under-actuation by foot rolling. For robots that are designed with this inspiration, such as point-feet walkers, walking motion is often analyzed in terms of limit cycles. Then, the local stability of the limit cycle can be studied by investigating deviations from nominal trajectories (gait sensitivity norm, ${ }^{1} H_{\infty}$ cost $^{3}{ }^{3}$ and L2 gain $^{4}$ ), or the speed of convergence back after such deviations (using Floquet theory ${ }^{5,6}$ ). The L2 gain calculation by Dai and Tedrake ${ }^{4}$ was successfully extended and implemented on a real robot, as recently documented by Griffin and Grizzle. ${ }^{7}$ Alternatively, the largest allowable terrain disturbance was maximized by Pratt et al., ${ }^{8}$ and trajectories were optimized to replicate human-walking data by Ames. ${ }^{9}$

Our approach to robustness quantification begins by stochastic modeling of the disturbances and (conservatively) defining what a failure is, e.g., slippage, scuffing, stance foot rotation, or a combination of such events. After discretizing the disturbance and state sets by meshing, step-to-step dynamics are studied to treat the system as a Markov chain. Then, the likelihood of failure can be easily quantified by calculating the expected number of steps before falling, or mean first-passage time (MFPT). ${ }^{10}$

An intuitive and capacious approach for controlling bipedal locomotion is to use two levels of control. Fixed low-level controllers are blind to environmental information, such as the terrain estimation. Optimizing the lowlevel controller for the expected number of steps before falling was previously impractical due to high computation time of MFPT for a given controller. However, our work over the years now allows us to estimate this number very quickly, and in turn, various low-level controllers can be optimized and benchmarked. Given environment

saglam@ece.ucsb.edu, katiebyl@ece.ucsb.edu 
and state information, the high-level control problem defines a policy to choose the right low-level controller at each step. This hierarchical approach provides even more dramatic improvements on the system performance.

The rest of this paper is organized as follows. We start by discretizing the walking dynamics in Section 2 to treat the system as a Markov chain. Then, we propose an intuitive and meaningful stability metric in Section 3. Using this metric, we then optimize and benchmark two controller schemes as case demonstrations in Section 4. A hierarchical control structure is adopted in Section 5 to increase the reliability of walking even more. Finally, the conclusion is presented in Section 6 .

\section{DISCRETIZATION OF STATES FOR MARKOV CHAIN REPRESENTATION}

Walking motions are governed by hybrid dynamics, and the discrete impacts when a foot comes into contact with the ground provide a natural discretization of the robot motion, which motivates studying step-to-step dynamics of walking. Assuming finite probabilities for significant terrain height disturbances being encountered while walking, underactuated bipedal robots are destined to fall. If the average failure rates are low (but nonzero), walking is metastable, as shown in Figure 1.

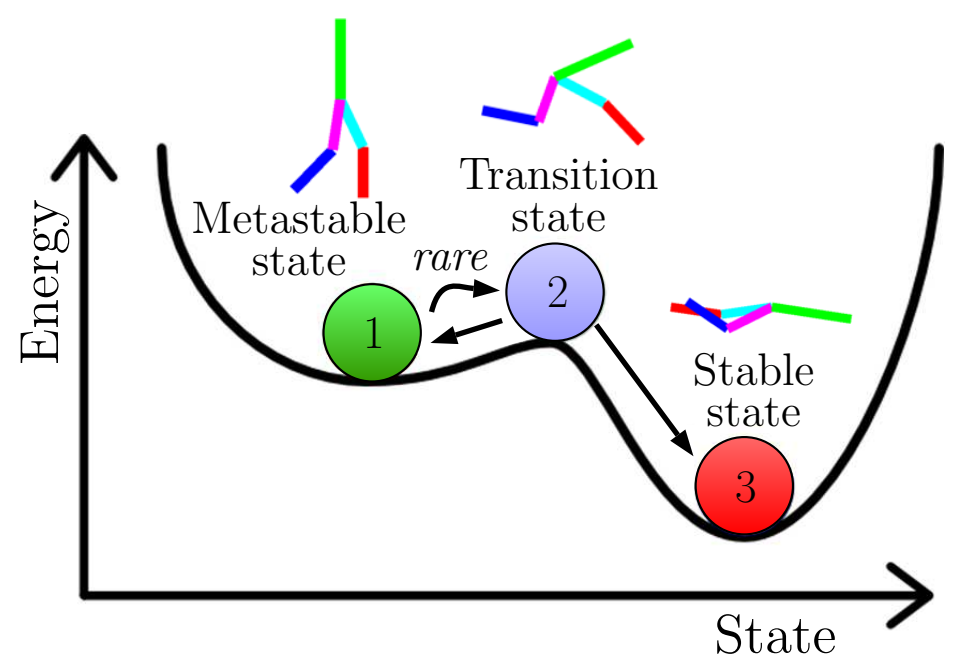

Figure 1: Starting from State 1, the probability of moving to absorbing State 3 goes to 1 over time, so State 1 is not stable in the strict sense. However, if the transitions from State 1 to State 2 are quite rare, then it is misleading to say State 1 is unstable. In this case State 1 is said to be metastable. In this representative figure, State 1, 2, and 3 correspond to walking, stumbling, and failure respectively.

Although walking is a "state" in the representative picture of Figure 1, the actual state of the robot is a vector of numeric values, e.g., of angles and velocities of the robot. So, the exact state of the robot will generally vary while walking, and our goal in discretization is to obtain finitely many states which well represent all the reachable configurations the robot may occupy. To illustrate, $\{0,0.01,0.02, \ldots, 1\}$ is a discretization of $[0,1]$.

In the discretization of states, the curse of dimensionality is a potential problem; however, given a walking controller and one-degree-of-freedom underactuation, the set of states which the robot may be found in at the end of a step turns out to be a quasi-2D manifold of the entire state space, ${ }^{11}$ which can be well-represented with a relatively low number of states for our purposes. Then, a stochastic model of the terrain leads naturally to a Markov chain representation of the walking motion.

\section{STABILITY METRIC DEVELOPMENT}

A Markov chain representation of walking lends itself to a very intuitive and easy quantification of reliability. When the concern is stability, the walking process can be approximated to consist of just two states as shown in Figure 2. As explained in a previous work, ${ }^{12}$ the walker visits states within a safe neighborhood within state space during walking on a given type of terrain. Within this neighborhood, it is then able to take the next step 
without falling with a probability very close to $\lambda_{2}$, which is the second largest eigenvalue of the transition matrix associated with the original Markov chain in hand. The remaining probability $\left(1-\lambda_{2}\right)$ maps the walking state to failure in the next step.

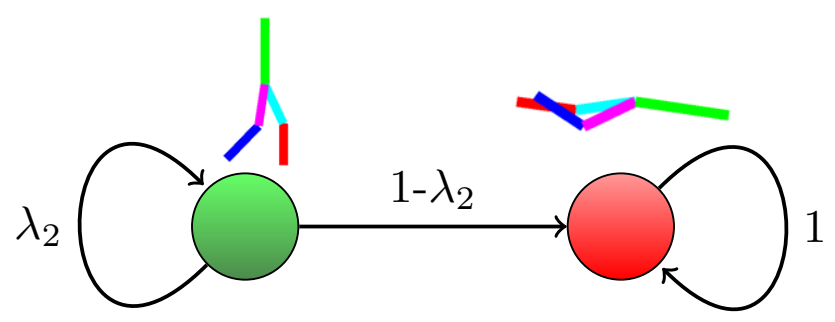

Figure 2: Approximation of the walking dynamics. With $\lambda_{2}$ probability, the robot takes the next step successfully, it fails otherwise. $\lambda_{2}$ is the second largest eigenvalue of the Markov chain.

The structure given in Figure 2 allows easily calculating

$$
\text { expected number of steps before falling }=\frac{1}{1-\lambda_{2}} .
$$

\section{OPTIMIZATION AND BENCHMARKING OF (LOW-LEVEL) CONTROL ACTION}

Using the quantification in (1), the performance of a walking controller can be optimized and benchmarked. While this method is applicable to any controller scheme, we study two particular control strategies as case demonstrations. One scheme is the now-familiar hybrid zero dynamics (HZD) approach and the other is a method using piece-wise reference trajectories with a sliding mode control (SMC).

\subsection{Optimization}

We first optimize the performance of the HZD control scheme to obtain the results in Figure 3a. As suggested in the seminal HZD paper, ${ }^{13}$ we first constrain speed to be $0.8 \mathrm{~m} / \mathrm{s}$ and optimize for energy. The performance of this strategy gives the red (bottom) line in Figure 3a. We then remove the speed constraint and minimize energy consumption, which does not provide much reliability improvement. We finally optimize the controller's stability by using

$$
\underset{\substack{\text { conimizler } \\
\text { parameters }}}{\operatorname{maximi}}\left\{\begin{array}{c}
\text { Expected } \\
\text { Number of Steps }
\end{array}\right\}=\underset{\substack{\text { controller } \\
\text { parameters }}}{\operatorname{maximi}}\left\{\frac{1}{1-\lambda_{2}}\right\} .
$$

Figure 3a shows that there is a great improvement in adopting this strategy. For the specific roughness marked on the figure, the robot is expected to take 113 steps instead of 6 when compared to the controllers optimized for energy.

To show the applicability of our method, we also optimize another controller scheme, which uses piece-wise reference trajectories with a sliding mode control (SMC) as formulated in previous work by the authors. ${ }^{14}$ One advantage of this controller scheme is that hand tuning the walking gait is relatively easy. Figure $3 \mathrm{~b}$ depicts the performance of a hand-tuned controller that was used in our previous studies. While optimizing for energy yields poor stability performance, the results in Figure 3b show great improvement in the robustness to terrain disturbance when the controller parameters are tuned using (2).

We note that Monte Carlo simulations are not a computationally practical means of verifying MFPT when it is very high, which has motivated our methodology throughout. However, we present a Monte Carlo study in Table 1 for $\mu=2$ and large enough $\sigma$ such that MFPT is small. To obtain the last column in this table, we simulated 10 thousand times. To allow the robot to "forget" the initial condition, we omit the first step, i.e., we only consider cases where it took more than a single step and do not count that first step. 


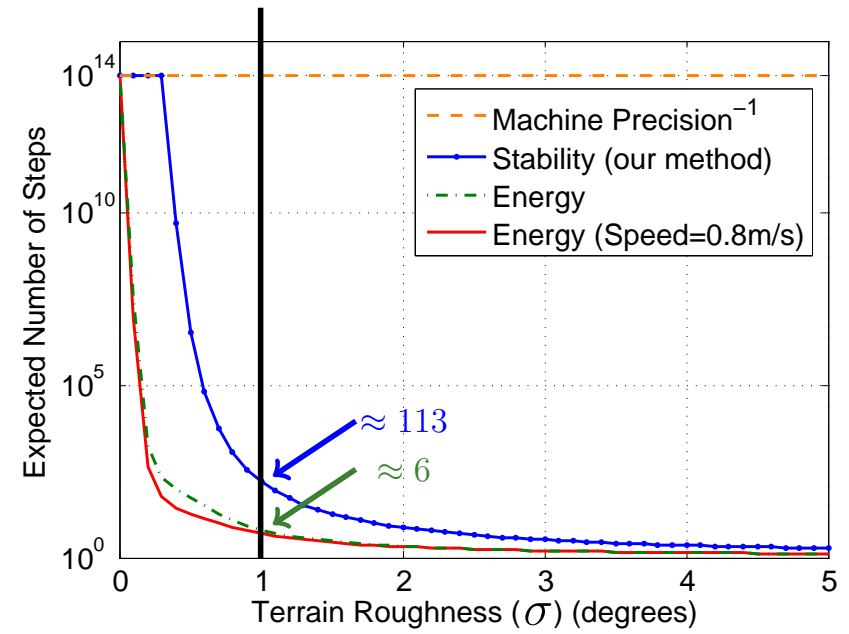

(a) Optimization of HZD control scheme.

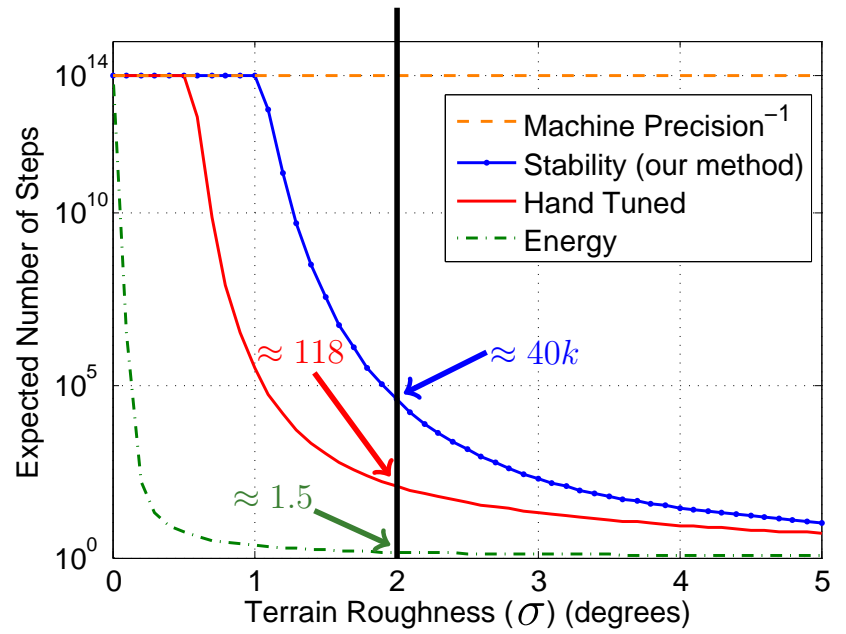

(b) Optimization of SMC control scheme.

Figure 3: Slopes ahead of the robot are assumed to be normally distributed with mean $\mu=0$ and standard deviation $\sigma$. Dashed line at $10^{14}$ steps is due to machine precision which corresponds to about a million tours around the world for a human-sized robot with half a meter step width.

Table 1: Verification of MFPT Estimation with Monte Carlo Simulations with $\mu_{s}=0$

\begin{tabular}{c|c|c|c|c}
$\begin{array}{c}\text { Terrain } \\
\text { Roughness }\end{array}$ & $\begin{array}{c}\text { Controller } \\
\text { Scheme }\end{array}$ & Optimized for & Estimation using (1) & Monte Carlo Simulation \\
\hline \multirow{3}{*}{$\sigma=2$} & \multirow{3}{*}{ HZD } & Energy (Speed=0.8m/s) & 2.2085 & 2.1511 \\
& & Energy & 2.2049 & 2.2487 \\
& & Stability & 5.5206 & 6.1290 \\
\hline \multirow{3}{*}{$\sigma=5$} & \multirow{2}{*}{ SMC } & Energy & 1.1470 & 1.5716 \\
& & Hand Tuned & 5.1766 & 5.0738 \\
& & Stability & 10.6433 & 10.4813
\end{tabular}




\subsection{Benchmark}

The second use of (1) is in comparing different controllers' performances. In Figure 4 we benchmark the performance of the HZD and SMC controller schemes when they are optimized for energy and stability. While the comparison in Figure 4b is not fair to the HZD controller, because it is originally designed for flat terrain, our goal is to illustrate the benchmarking capability of our performance quantification.

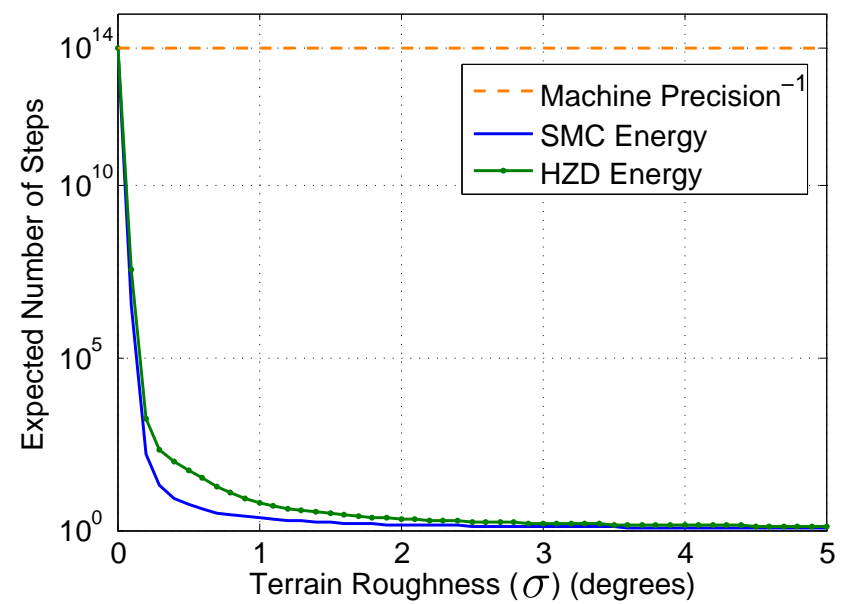

(a) Comparison of HZD and SMC controllers optimized for energy efficiency.

Figure 4: Benchmarking of optimized HZD and SMC control schemes. Slopes ahead of the robot are assumed to be normally distributed with mean $\mu=0$ and standard deviation $\sigma$. Dashed line at $10^{14}$ steps is due to machine precision which corresponds to about a million tours around the world for a human-sized robot with half a meter step width.

\section{HIGH-LEVEL CONTROL DESIGN}

One could easily imagine different controllers having various advantages under a variety of conditions, e.g., some controllers might walk better uphill, some may achieve the speed we desired, some may be more energy efficient, and others may have the step width we need. These controllers might be designed using different controller schemes and they can be optimized for different cost functions. A very intuitive idea at this point is to adopt a hierarchical control structure as shown in Figure 5a. The high-level controller then choses the optimal low-level controller at each step using noisy terrain estimation and state information.

Optimal high-level behavioral algorithms, e.g., lean the torso forward when going uphill, are obtained using dynamic programming ${ }^{15}$ on the Markov decision process model of the system. Figure $5 \mathrm{~b}$ shows the resulting performance of adopting such a high-level control. ${ }^{16}$

\section{CONCLUSION}

In this paper we presented a complete approach to the robust control of bipedal locomotion. For reliable operation under disturbances like rough terrain, measuring the stability of the system is essential. By quantifying the failure rates by the expected number of steps, we optimize and benchmark any given controller scheme. We finally presented the dramatic improvements gained by adopting a hierarchical control structure. The general approach presented in this paper is applicable to a variety of systems including numerous legged robots.

\section{ACKNOWLEDGMENT}

This work is supported by the Institute for Collaborative Biotechnologies through grant W911NF-09-0001 from the U.S. Army Research Office. The content of the information does not necessarily reflect the position or the policy of the Government, and no official endorsement should be inferred. 

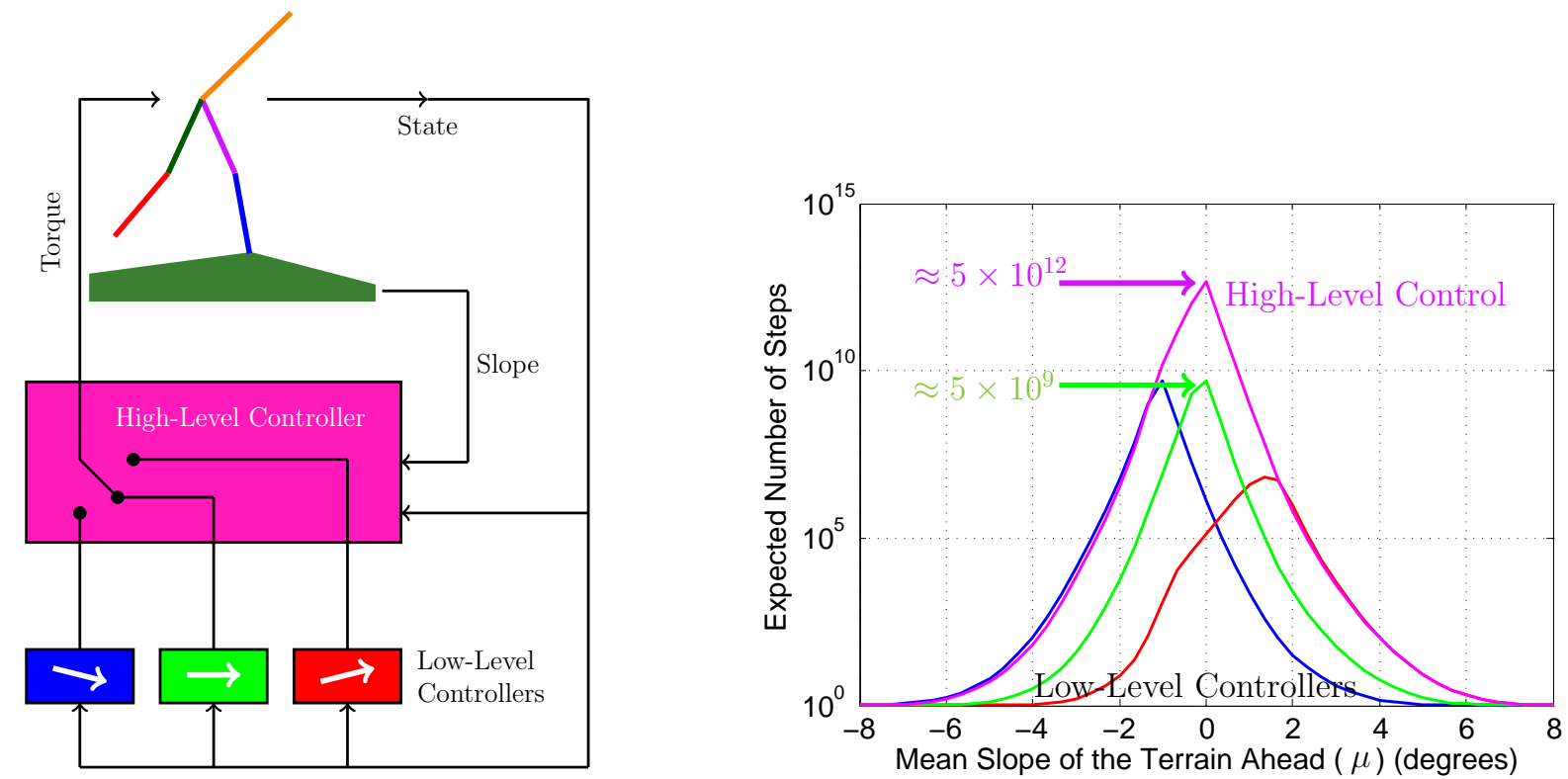

(a) Hierarchical control structure. In this example there are (b) The improvement of adopting a high-level controller three low-level controllers available. As shown in Figure 5b drawn in Figure 5a. Slopes ahead of the robot are assumed to blue, green, and red controllers work better when it is down- be normally distributed with mean $\mu$ and standard deviation hill, flat, and uphill respectively. $\sigma=1$.

Figure 5: The high-level controller choses the right low-level controller at each step using the optional noisy terrain estimation and state information.

\section{REFERENCES}

[1] Hobbelen, D. and Wisse, M., "A Disturbance Rejection Measure for Limit Cycle Walkers: The Gait Sensitivity Norm," IEEE Transactions on Robotics 23, 1213-1224 (Dec. 2007).

[2] Vukobratovic, M. and Borovac, B., "Zero-moment point - thirty five years of its life," International Journal of Humanoid Robotics 1(01), 157-173 (2004).

[3] Morimioto, J., Zeglin, G., and Atkeson, C., "Minimax differential dynamic programming: application to a biped walking robot," in [SICE 2003 Annual Conference], 3, 2310-2315 Vol.3 (Aug. 2003).

[4] Dai, H. and Tedrake, R., "L2-gain optimization for robust bipedal walking on unknown terrain," in [Robotics and Automation (ICRA), 2013 IEEE International Conference on], 3116-3123, IEEE (2013).

[5] Hurmuzlu, Y. and Basdogan, C., "On the Measurement of Dynamic Stability of Human Locomotion," Journal of Biomechanical Engineering 116, 30-36 (Feb. 1994).

[6] McGeer, T., "Passive Dynamic Walking," The International Journal of Robotics Research 9, 62-82 (Apr. 1990).

[7] Griffin, B. and Grizzle, J., "Walking Gait Optimization for Accommodation of Unknown Terrain Height Variations," (2015). Submitted.

[8] Pratt, J., Chew, C.-M., Torres, A., Dilworth, P., and Pratt, G., "Virtual Model Control: An Intuitive Approach for Bipedal Locomotion," The International Journal of Robotics Research 20, 129-143 (Feb. 2001).

[9] Ames, A. D., "First Steps toward Automatically Generating Bipedal Robotic Walking from Human Data," in [Robot Motion and Control 2011], Kozowski, K., ed., Lecture Notes in Control and Information Sciences, 89-116, Springer London (Jan. 2012).

[10] Byl, K. and Tedrake, R., "Metastable Walking Machines," The International Journal of Robotics Research 28, 1040-1064 (Aug. 2009). 
[11] Saglam, C. O. and Byl, K., "Stability and gait transition of the five-link biped on stochastically rough terrain using a discrete set of sliding mode controllers," in [IEEE International Conference on Robotics and Automation (ICRA)], 5675-5682 (May 2013).

[12] Saglam, C. O. and Byl, K., "Metastable Markov Chains," in [IEEE Conference on Decision and Control $(C D C)],($ Dec. 2014).

[13] Westervelt, E., Grizzle, J. W., and Koditschek, D., "Hybrid zero dynamics of planar biped walkers," IEEE Transactions on Automatic Control 48, 42-56 (Jan. 2003).

[14] Saglam, C. O. and Byl, K., "Switching policies for metastable walking," in [Proc. of IEEE Conference on Decision and Control (CDC)], 977-983 (Dec. 2013).

[15] Bellman, R., "A Markovian decision process," Indiana University Mathematics Journal 6(4), 679-684 (1957).

[16] Saglam, C. O. and Byl, K., "Robust Policies via Meshing for Metastable Rough Terrain Walking," in [Proceedings of Robotics: Science and Systems], (2014). 